\title{
Commentaries
}

\section{The pathophysiology of autoimmune blistering diseases}

\author{
Kim B. Yancey \\ Department of Dermatology, Medical College of Wisconsin, Milwaukee, Wisconsin, USA.
}

\begin{abstract}
Knowledge of the pathophysiology of immunobullous diseases has been advanced by the demonstration that passive transfer of antibodies against skin autoantigens can induce blisters in experimental animals with clinical, histologic, and immunopathologic features similar to those seen in human patients. In this issue of the JCI, Liu et al. extend their earlier observations regarding an experimental murine model of bullous pemphigoid by showing that the plasminogen/plasmin signaling cascade synergizes with MMP-9 during the early phase of antibody-induced blister formation in vivo (see the related article beginning on page 879). In a separate study, Sitaru et al. show for the first time to my knowledge that passive transfer of experimental antibodies against type VII collagen create subepidermal blisters in mice that mimic those seen in patients with epidermolysis bullosa acquisita (see the related article beginning on page 870 ). While the articles by Liu, Sitaru, and their colleagues identify pathways of inflammation and tissue injury that, if interrupted, may abrogate blister formation, in a third study, Payne et al. utilized phage display technologies to isolate human anti-desmoglein monoclonal antibodies from a patient with pemphigus vulgaris and show that such antibodies have restricted patterns of heavy and light chain gene usage - findings suggesting that autoantibodies may represent an additional target for therapeutic interventions in patients with immunobullous diseases (see the related article beginning on page 888 ).
\end{abstract}

The stratified squamous epithelium of the human epidermis forms a continuous barrier against the external environment. The pathophysiology of blistering diseases illustrates how impairments in epithelial adhesion lead to disorders characterized by substantial morbidity and/or mortality. Blistering diseases can be inherited or acquired; most examples of the latter are autoimmune in nature and are characterized by autoantibodies that target adhesion junctions promoting either cell-cell or cell-matrix adhesion in skin. Patients with pemphigus, a family of intraepidermal autoimmune blistering diseases, have autoantibodies that target cadherins (specifically, desmogleins) in desmosomes, adhesion junctions that anchor the intermediate filament cytoskeleton to keratinocyte plasma membranes at

Nonstandard abbreviations used: BM, basement membrane; BP, bullous pemphigoid; BPAG, BP antigen; EBA, epidermolysis bullosa acquisita.

Conflict of interest: The author has declared that no conflict of interest exists.

Citation for this article: J. Clin. Invest. 115:825-828 (2005). doi:10.1172/JCI200524855. cell-cell borders (1). Patients with bullous pemphigoid (BP) and other autoimmune subepidermal blistering diseases have autoantibodies that target autoantigens in epidermal basement membrane (BM) (2, 3 ). The major ultrastructural subregions of epidermal BM include the intermediate filament cytoskeleton, hemidesmosomes, and plasma membranes of basal keratinocytes; the transmembrane elements of hemidesmosomes and associated anchoring filament complexes within the lamina lucida; the lamina densa (i.e., BM proper); and the underlying sublamina densa region, containing anchoring fibrils and fibrillar proteins of the papillary dermis (Figure 1) (4). Translational research over the past 25 years has demonstrated that patients with pemphigus, BP, and other autoimmune blistering diseases have autoantibodies that target specific antigens in skin; that such autoantigens often represent components of adhesion junctions; and that mutations in genes encoding such proteins are responsible for inherited diseases characterized by skin fragility, blister formation, and/or ectodermal dysplasia (Table 1).

\section{Subepidermal immunobullous diseases}

In this issue of the JCI, 2 articles describe how passive transfer of experimental IgG directed against murine homologs of 2 human epidermal BM collagens, BP180 (also known as BP antigen 2 [BPAG2] or type XVII collagen) and type VII collagen, was used to develop animal models of BP (5) and epidermolysis bullosa acquisita (EBA) (6), respectively.

\section{Bullous pemphigoid}

BP is a chronic subepidermal blistering disease typically seen in the elderly $(2,3)$. Though BP is a polymorphic skin disease, lesions usually consist of tense blisters situated on either inflamed or noninflamed skin; pruritus may be severe or nonexistent. Biopsies of lesional skin show subepidermal blisters that are either granulocyterich or granulocyte-poor, depending on whether the biopsies were obtained from inflamed or noninflamed skin. Direct immunofluorescence microscopy of perilesional skin shows linear deposits of IgG and/ or complement component C3 in epidermal BM. Patients with BP have circulating IgG autoantibodies against 2 hemidesmosome proteins, BP230 (also known as BPAG1) and BP180. BP230 is a plakin protein family member that promotes the association of hemidesmosomes with keratin intermediate filaments. BP180 is a type II, transmembrane collagen that is associated with hemidesmosome-anchoring filament complexes and is thought to harbor the pathogenic epitope responsible for the initiation of $\mathrm{BP}(7)$. The extracellular domain of this protein contains 15 interrupted collagenous domains. Rotary shadowing studies of purified BP180 image its intracytoplasmic region as a globular head and its ectodomain as a central rod joined to a flexible tail (7). Immunoelectron microscopy studies indicate that BP180 spans the lamina lucida and inserts into the lamina densa $(8,9)$. BP180 is targeted by autoantibodies from patients with BP, pemphigoid gestationis, cicatricial pemphigoid, and linear IgA dermatosis $(2,3)$. 


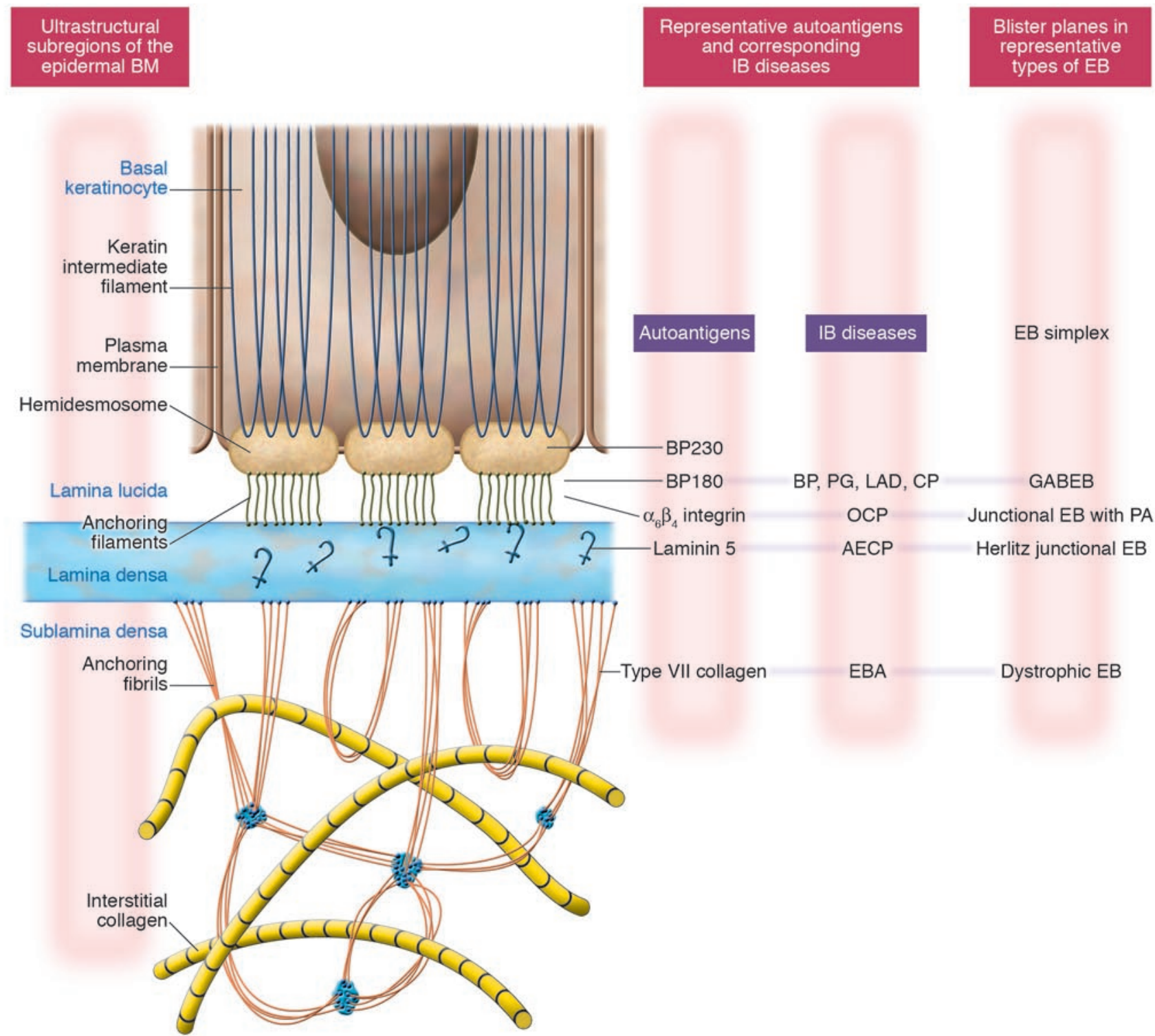

Figure 1

Schematic model of the epidermal BM. The major subregions of epidermal BM are depicted in the context of autoimmune and genetic blistering diseases that develop as a consequence of acquired or inherited impairments in proteins within this cell-matrix adhesion junction. AECP, antiepiligrin cicatricial pemphigoid; CP, cicatricial pemphigoid; EB, epidermolysis bullosa; IB, immunobullous; LAD, linear IgA dermatosis; OCP, ocular cicatricial pemphigoid. GABEB, generalized atrophic benign epidermolysis bullosa; PA, pyloric atresia.

Epitope mapping studies of recombinant proteins have previously shown that autoantibodies from most patients with $\mathrm{BP}$ bind a determinant within the sixteenth noncollagenous domain of BP180 (i.e., the portion of its ectodomain that is positioned adjacent to plasma membranes of basal keratinocytes) (10). Passive transfer of experimental IgG developed against the murine homolog of this determinant to neonatal $\mathrm{BALB} / \mathrm{c}$ mice produces clinical, histologic, and immunopathologic alterations like those seen in patients with BP (11). Antibody-induced blister formation in this animal model is dependent upon the activation of complement, degranulation of dermal mast cells, and generation of neutrophil-rich infiltrates (11-16). The finding that MMP-9-deficient mice are resistant to anti-BP180 IgG (14) has been explained by the observation that neutrophil-derived MMP-9 inactivates $\alpha 1$-proteinase inhibitor, which allows unrestrained activity of neutrophil elastase that degrades BP180 and produces subepidermal blisters in this experimental model (Figure 2) (16). In this issue of the JCI, Liu and colleagues show that MMP-9 activation in this murine model of BP is plasmin dependent and that plasmin activates MMP-9 independent of MMP-3 (5). While antibody-induced blister formation was restrained by plasmin inhibition with $\alpha 2$-antiplasmin for 12 hours following the administration of pathogenic IgG, studies in mice genetically engineered to lack plasminogen or both tissue and urokinase plasminogen activators found that plasmin-independent pathways activated MMP-9 and yielded subepidermal blisters within 24 hours. Whether MMP-9 inhibition can be used to restrain proinflammatory mechanisms in BP before 


\section{Table 1}

Diseases of skin fragility, dysplasia, and/or blistering

$\begin{array}{lc}\text { Adhesion type } & \text { Structural target } \\ \text { Cell-cell } & \text { Desmosomes }\end{array}$

Cell-matrix

\author{
Protein target \\ Dsg 1, 3, and 4; \\ Plakophilin I; \\ Plakoglobin; \\ Desmoplakins \\ BP180 and BP230 \\ $\alpha_{6} \beta_{4}$ integrin \\ Laminin 5 \\ Type VII collagen
}
Autoimmune diseases
PV, PF, PNP

BP, PG, LAD, CP
OCP
AECP
EBA

Genetic diseases

Ectodermal dysplasias, skin fragility

syndromes, altered epidermal differentiation
Epidermolysis bullosa

AECP, anti-epiligrin cicatricial pemphigoid; CP, cicatricial pemphigoid; Dsg, desmoglein; LAD, linear IgA dermatosis; OCP, ocular cicatricial pemphigoid; PF, pemphigus foliaceus; PG, pemphigoid gestationis; PNP, paraneoplastic pemphigus; PV, pemphigus vulgaris.

complementary mechanisms supervene remains to be determined.

\section{Epidermolysis bullosa acquisita}

Once considered to represent a diagnosis of exclusion or a group of heterogeneous blistering disorders, EBA is now recognized as a polymorphic, yet distinct, subepidermal blistering disease (3). Patients with the dermolytic, or noninflammatory, variant of EBA have trauma-induced blisters and erosions on noninflamed skin, atrophic scars, milia, nail dystrophy, and/or oral erosions. Other patients have widespread inflammatory blisters that mimic those seen in patients with BP. Some patients transition from the inflammatory to the dermolytic form of the disease. EBA is typically chronic and resistant to treatment; many patients also have underlying inflammatory bowel disease. EBA was shown to represent an autoimmune disease by the demonstration that such patients possess in situ and circulating autoantibodies against the sublamina densa region of the epidermal BM (Figure 1) (3). Autoantibodies from EBA patients were used to identify a specific autoantigen in dermal extracts that was initially termed the EBA antigen (17). Subsequent studies showed that this autoantigen was in fact type VII collagen (18). Type VII collagen is unique to stratified squamous epithelium; it consists of 3 identical $\alpha$ chains, each with a molecular weight of $290 \mathrm{kDa}$. The amino terminus of type VII collagen contains a large globular noncollagenous domain termed NC1; a small noncollagenous domain termed NC2 lies at its carboxyl terminus. Anchoring fibrils are formed as a consequence of antiparallel alignment of individual type VII collagen molecules that subsequently unite via disulfide bonds within their NC2 tails. Once a tail-to-tail dimer is formed, the NC2 domain is proteolytically cleaved, leaving a long, thread-like macromolecule characterized by a central rod with large, globular NC1 domains at each end. Type VII collagen dimers then aggregate laterally to form anchoring fibrils (Figure 1), which create a network of looping bands along the inferior aspect of the lamina densa that serve as a tether for dermal fibrillar proteins (19).

In this issue of the JCI, Sitaru and colleagues passively transferred rabbit IgG developed against murine type VII collagen NC1 recombinant peptides into adult nude, $\mathrm{BALB} / \mathrm{c}$, and C57BL/ 6 mice, thereby creating subepidermal blisters that clinically, histologically, and immunopathologically resemble those seen in patients with EBA (6). Lesion formation in this animal model was complement dependent; interestingly, only those neonatal mice that received concordant intradermal injections of neutrophils, complement component C5a, and IL-8 developed lesions. The demonstration that anti-type VII collagen $\operatorname{IgG}$ is pathogenic (i.e., capable of inducing subepidermal blisters) in vivo represents a noteworthy advance in our understanding of this disease. Specific questions raised by this study include the following: (a) why were such large doses of high titer IgG required for induction of blisters in this animal model (whereas most EBA patients have low titers of circulating anti-BM IgG)? (b) what accounted for the relative resistance of neonatal mice to antitype VII collagen IgG (most passive transfer animal models utilize newborn mice due to their increased susceptibility to blister formation)? (c) what was the phenotype of the animals that harbored anti-type VII collagen IgG in the epidermal BM for prolonged periods (i.e., did such mice display a dermolytic, EBA phenotype)? (d) how did local factors (e.g., pressure, trauma, and thermal influences) contribute to lesion formation? and (e) what accounted for weight loss in mice that received pathogenic IgG, given their absence of clinical or histologic alterations in oral, esophageal, or colonic epithelia?

\section{Future directions: autoantibodies as targets for disease intervention}

In this issue of the JCI, Payne and colleagues utilized phage display technologies to isolate pathogenic (i.e., blister-inducing) and nonpathogenic human anti-desmoglein monoclonal antibodies as single-chain variable region fragments from a patient with

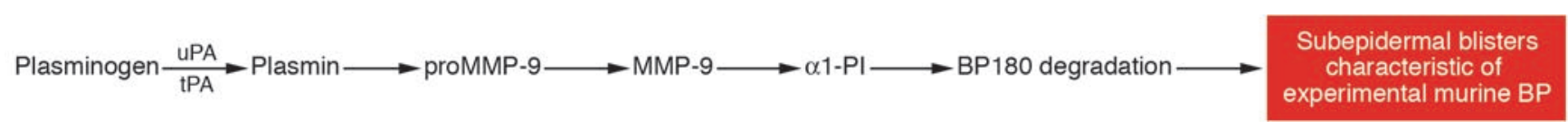

Figure 2

Schematic overview of the plasminogen/plasmin activation cascade in experimental murine BP. Anti-BP180 IgG binds epidermal BM, activates complement, and generates neutrophil-rich infiltrates in skin. Subsequently, plasmin activates MMP-9, which in turn inactivates $\alpha 1-$ proteinase inhibitor $(\alpha 1-\mathrm{PI})$, thus allowing unrestrained activity of neutrophil elastase that degrades BP180 and produces subepidermal blisters. tPA, tissue plasminogen activator; uPA, urokinase plasminogen activator. 
active mucocutaneous pemphigus vulgaris (20). Inhibition ELISAs showed that epitopes defined by these monoclonal antibodies are blocked by autoantibodies from multiple patients with pemphigus. Moreover, genetic analysis of these antibodies revealed restricted patterns of heavy- and light-chain gene utilization. Taken together, these findings suggest several novel approaches to abrogating disease (e.g., targeting $B$ cells that express certain $V_{H}$ gene segments, generating peptides that may block pathogenic antibodies reactive with shared and dominant epitopes, or using blocking anti-idiotypic antibodies). These approaches, coupled with better understanding of the contributions of molecular mimicry as well as mechanisms of central and peripheral tolerance to disease pathogenesis, will facilitate the development of effective interventions that counteract the chronic morbidity (i.e., pruritus, blistering, infection, scarring, and treatment-related immunosuppression) and, at times, mortality of immunobullous diseases.

\section{Acknowledgments}

This work was supported in part by a grant from the NIH (RO1 AR048982-01A1) to the author.
Address correspondence to: Kim B. Yancey, Medical College of Wisconsin, Department of Dermatology, 8701 Watertown Plank Road, Milwaukee, Wisconsin 53226-4801, USA. Phone: (414) 456-4081; Fax: (414) 456-6518; E-mail: kyancey@mcw.edu.

1. Udey, M.C., and Stanley, J.R. 1999. Pemphigus-diseases of antidesmosomal autoimmunity. JAMA. 282:572-576

2. Yancey, K.B., and Egan, C.A. 2000. Pemphigoid: clinical, histologic, immunopathologic, and therapeutic considerations. JAMA. 284:350-356.

3. Schmidt, E., and Zillikens, D. 2000. Autoimmune and inherited subepidermal blistering diseases: advances in the clinic and the laboratory. Adv. Dermatol. 16:113-157.

4. Burgeson, R.E., and Christiano, A.M. 1997. The dermal-epidermal junction. Curr. Opin. Cell Biol. 9:651-658.

5. Liu, Z., et al. 2005. Synergy between a plasminogen cascade and MMP-9 in autoimmune disease. J. Clin. Invest. 115:879-887. doi:10.1172/JCI200523977.

6. Sitaru, C., et al. 2005. Induction of dermal-epidermal separation in mice by passive transfer of antibodies to type VII collagen. J. Clin. Invest. 115:870-878. doi:10.1172/JCI200521386.

7. Van den Bergh, F., and Giudice, G.J. 2003. BP180 (type XVII collagen) and its role in cutaneous biology and disease. Adv. Dermatol. 19:37-71.

8. Masunaga, T., et al. 1997. The extracellular domain of BPAG2 localizes to anchoring filaments and its carboxyl terminus extends to the lamina densa of normal human epidermal basement membrane. J. Invest. Dermatol. 109:200-206.

9. Nonaka, S., et al. 2000. The extracellular domain of BPAG2 has a loop structure in the carboxy terminal flexible tail in vivo. J. Invest. Dermatol. 115:889-892.

10. Giudice, G.J., et al. 1993. Bullous pemphigoid and herpes gestationis autoantibodies recognize a common non-collagenous site on the BP180 ectodomain. J. Immunol. 151:5742-5750.

11. Liu, Z., et al. 1993. A passive transfer model of the organ-specific autoimmune disease, bullous pemphigoid, using antibodies generated against the hemidesmosomal antigen, BP180. J. Clin. Invest. 92:2480-2488

12. Liu, Z., et al. 1995. The role of complement in experimental bullous pemphigoid. J. Clin. Invest. 95:1539-1544.

13. Liu, Z., et al. 1997. A major role for neutrophils in experimental bullous pemphigoid. J. Clin. Invest. 100:1256-1263.

14. Liu, Z., et al. 1998. Gelatinase B-deficient mice are resistant to experimental bullous pemphigoid. J. Exp. Med. 188:475-482.

15. Liu, Z., et al. 2000. A critical role for neutrophil elastase in experimental bullous pemphigoid. J. Clin. Invest. 105:113-123.

16. Liu, Z., et al. 2000. The serpin alpha1-proteinase inhibitor is a critical substrate for gelatinase B/ MMP-9 in vivo. Cell. 102:647-655.

17. Woodley, D.T., et al. 1984. Identification of the skin basement-membrane autoantigen in epidermolysis bullosa acquisita. N. Engl. J. Med. 310:1007-1013.

18. Woodley, D.T., et al. 1988. Epidermolysis bullosa acquisita antigen is the globular carboxyl terminus of type VII procollagen. J. Clin. Invest. 81:683-687.

19. Shimizu, H., et al. 1997. Most anchoring fibrils in human skin originate and terminate in the lamina densa. Lab. Invest. 76:753-763.

20. Payne, A.S., et al. 2005. Genetic and functional characterization of human pemphigus vulgaris monoclonal autoantibodies isolated by phage display. J. Clin. Invest. 115:888-899. doi:10.1172/ JCI200524185.

\title{
To live or die: a critical decision for the lung
}

\author{
G.R. Scott Budinger and Jacob I. Sznajder
}

Division of Pulmonary and Critical Care Medicine, Northwestern University, Chicago, Illinois, USA.

\begin{abstract}
Every cell in the body expresses a set of proteins designed to trigger permeabilization of the mitochondria and cell death. Inactivation or inappropriate triggering of these pathways is increasingly recognized as a contributor to human disease. A study in this issue of the JCI demonstrates that IL-6 exerts its protective effect against the development of lung injury following exposure of mice to $95 \% \mathrm{O}_{2}$ by increasing the expression of a Bcl-2-related protein, $\mathrm{A1}$ (see the related article beginning on page 1039). This protein acts to prevent mitochondrial membrane permeabilization and cell death following exposure to hyperoxia. The data in this study lend support to the hypothesis that inappropriate triggering of cell-death pathways may contribute to the development of hyperoxic pulmonary edema, lung injury, and respiratory failure.
\end{abstract}

To maintain homeostasis, cell growth must be precisely balanced by cell death. It is there-

Nonstandard abbreviations used: $\mathrm{BH}, \mathrm{Bcl}-2$ homology; KGF, keratinocyte growth factor.

Conflict of interest: The authors have declared that no conflict of interest exists.

Citation for this article: J. Clin. Invest. 115:828-830 (2005). doi:10.1172/JCI200524681. fore not surprising that long-lived mammals have highly conserved, complex pathways that regulate cell death. The importance of these pathways has been demonstrated during embryogenesis and in many pathologic conditions (1). Disturbance of the cell-death pathways can result in either malignancy or autoimmune disorders, while premature cell death has been implicated in the devel- opment of degenerative diseases, immunodeficiency, and infertility (1). Studies of the apoptotic pathways in the past decade have led to the realization that, at a molecular level, cells in the body are poised to "sacrifice" themselves for the benefit of the organism. This has led some investigators to speculate that inappropriate activation of these pathways may explain the convergence of a number of different injurious stimuli in the phenotypes of either lung injury or multiple organ dysfunction syndrome (2).

In many nonimmune mammalian cells, death pathways converge at the mitochondria, which serve a dual role as the primary metabolic engines of the cell and as critical cell-death checkpoints. As depicted schematically in Figure 1, exposure to a death signal results in the permeabilization of the mitochondrial outer membrane, releasing a variety of proteins, including cytochrome $c$, 